Short Communication

\title{
Estimation of rate constants of drug binding to open channels of cardiac transient outward current
}

\author{
Jiř́ Šimurda and Milena Šimurdová \\ Department of Physiology, Faculty of Medicine, Masaryk University, Brno, Czech Republic
}

\begin{abstract}
An improved approach to the evaluation of the rate constants of drugs binding to the open channel underlying the transient outward potassium current $I_{t o}$ is described. It is based on an analysis of a quantitative model formulated by a set of twelve differential equations. The rate constants are calculated from the time constants resulting from an approximation of the time course of apparent inactivation of the recorded $I_{t o}$ by two exponentials in the absence and by three exponentials in the presence of a blocking agent. The model study confirmed significantly higher accuracy in comparison with the existing electrophysiological method.
\end{abstract}

Key words: $I_{t o}$ channel - Drug binding - Open state - Rate constants - Mathematical model

\begin{abstract}
Abbreviations: $\alpha_{q}, \beta_{q}$, rate constants of activation; $\alpha_{r}, \beta_{r}$, rate constants of fast inactivation; $\alpha_{s}, \beta_{s}$, rate constants of slow inactivation; $[D]$, drug concentration; $I_{t o}$, transient outward current; $k_{o n}$, $k_{\text {off }}$, rate constants of drug binding; Kv4.3, a phenotype of $I_{t o}$ channel; $\tau_{b f}, \tau_{b s}, \tau_{b u s}$, fast, slow and ultraslow time constants of apparent inactivation in the presence of a drug; $\tau_{i}, \tau_{i}$, $\tau_{i}$, time constants of inactivation; $V$, membrane voltage.
\end{abstract}

Transient outward potassium current $\left(I_{t o}\right)$ plays a key role in early repolarization of the cardiac action voltage from many species, including man. Many electrophysiological, genetic, and crystal structure studies have been done to reveal the characteristic features of channel gating (for review, see Patel and Campbell 2005). A variety of commonly used therapeutic agents as antiarrhythmic, antidepresant, and antimalarial drugs display $I_{t o}$ channel blocking properties (e.g. Jahnel et al. 1994; Clark et al. 1995; Wettwer et al. 1998; Casis and Sanchez-Chapula 1998; Sanchez-Chapula 1999; Bébarová et al. 2005; Bébarová et al. 2009; Wagner et al. 2010). Mechanisms of drug-channel interactions are known to depend on channel state (Hille 1977). Most blockers of $I_{t o}$ are thought to bind to their receptor only when the channels are in the open state. Block in closed state (Castle and Slawsky 1993) or in two states (open and closed (Christé et al. 1995) or open and inactivated (Caballero et al. 2003)) is less commonly anticipated.

Correspondence to: Jiří Šimurda, Department of Physiology, Faculty of Medicine, Masaryk University, Kamenice 5, 62500 Brno-Bohunice, Czech Republic

E-mail: simurda@med.muni.cz
One remarkable feature of the open channel block is the accelerated apparent inactivation of $I_{t o}$ (e.g. Jahnel et al. 1994; Clark et al. 1995; Casis and Sánchez-Chapula 1998; Wettwer et al. 1998; Sanchez-Chapula 1999; Bébarová et al. 2005; 2009; Wagner et al. 2010). In the case of monoexponential inactivation in the absence of a drug, the apparent inactivation becomes biexponential (showing a fast and a slow component) in the presence of a drug. The early acceleration can be explained as simultaneous inactivation and block of the open channel. The slow component corresponds to the slower transition of the blocked channels through the open to the inactivated states. In addition, at least two components of inactivation have been identified for the known phenotypes of $I_{t o}$ (Patel et al. 2005) so that three or more exponential processes can be distinguished in the presence of the drug effect.

In this study we addressed the question of the precision with which the association and dissociation rate constants can be assessed from the $I_{t o}$ currents recorded at different concentrations of a blocking agent. To investigate the open channel block, we supplemented a mathematical model of the gating mechanism of the $I_{t o}$ channel described by Patel et al. (2004) with a transition of the open channel to the blocked state. The activation-independent inactivation gate is regarded as being 
responsible for fast inactivation while the slow inactivation (and the block) is thought to occur only from the open state (Fig. 1A). In the study of Patel et al. (2004), the rate constants of gating are related to Kv4.3 channels expressed in Xenopus oocytes. In an effort to relate the model to the results measured in native channels e.g. in rat ventricular myocytes (Jahnel et al. 1994; Bébarová et al. 2005, 2009) we modified the voltage dependence of the rate constants of gating as follows:

$$
\begin{aligned}
\alpha_{q} & =-2000 /(1+\exp ((V-57) / 24))+2000, \\
\beta_{q} & =120 /(1+\exp ((V+47) / 16)), \\
\alpha_{r} & =41.4 /(1+\exp ((V+84) / 10)), \\
\beta_{r} & =28.8 /(1+\exp (-(V+20) / 15)), \\
\alpha_{s} & =8 \mathrm{~s}^{-1}, \beta_{s}=12 \mathrm{~s}^{-1},
\end{aligned}
$$

where $V$ is membrane voltage. The rate constants of slow inactivation are regarded as being voltage independent.

We aimed to explore the dependence of characteristic features of open state channel block on the kinetics of drugchannel interactions described by dissociation and association rate constants $k_{o f f}, k_{o n}$ and eventually on the rate constants of slow inactivation $\alpha_{s}, \beta_{s}$. Provided that the current responses to depolarizing steps to positive values of membrane voltage ensuring fast and full activation were evaluated, most results of simulations appeared to be only slightly dependent on numerical values of kinetic parameters of gating.

Figure 1A depicts transitions among channel states described by a set of 12 differential equations that were solved in two ways to ensure accuracy: (i) analytically as a set of linear differential equations with constant coefficients using the program MATLAB 6.5 (The MathWorks, Inc.) and (ii) numerically using the solver ODE15s in the MATLAB.

Figure1B shows the time courses of the fraction of open channels in response to a single voltage step from -120 to $+60 \mathrm{mV}$ applied at different drug concentrations $\left(k_{\text {off }}=20\right.$ $\mathrm{s}^{-1}$ and $k_{o n}[D]$ in the range $\left.0-100 \mathrm{~s}^{-1}\right)$. The drug-induced acceleration of apparent inactivation is evident. Panels $C$ and $\mathrm{D}$ in Fig. 1 display superimposed simultaneous time courses of fractions of channels in particular states in response to the same voltage step at $k_{o n}[D]=k_{\text {off }}=20 \mathrm{~s}^{-1}$. At $-120 \mathrm{mV}$ practically all channels are found in the closed states $C_{1}$ and $C_{2}$. During the first $3 \mathrm{~ms}$ channels pass through the closed states $\mathrm{C}_{2}-\mathrm{C}_{4}$ to the open state $\mathrm{O}$ (Fig. 1C) and subsequently to the inactivated states $\mathrm{I}_{\text {fast }}, \mathrm{I}_{\text {slow, }}$ and the blocked state B (Fig. 1D). Finally, practically all channels transit to the inactivated state $\mathrm{I}_{\text {fast }}$.

A
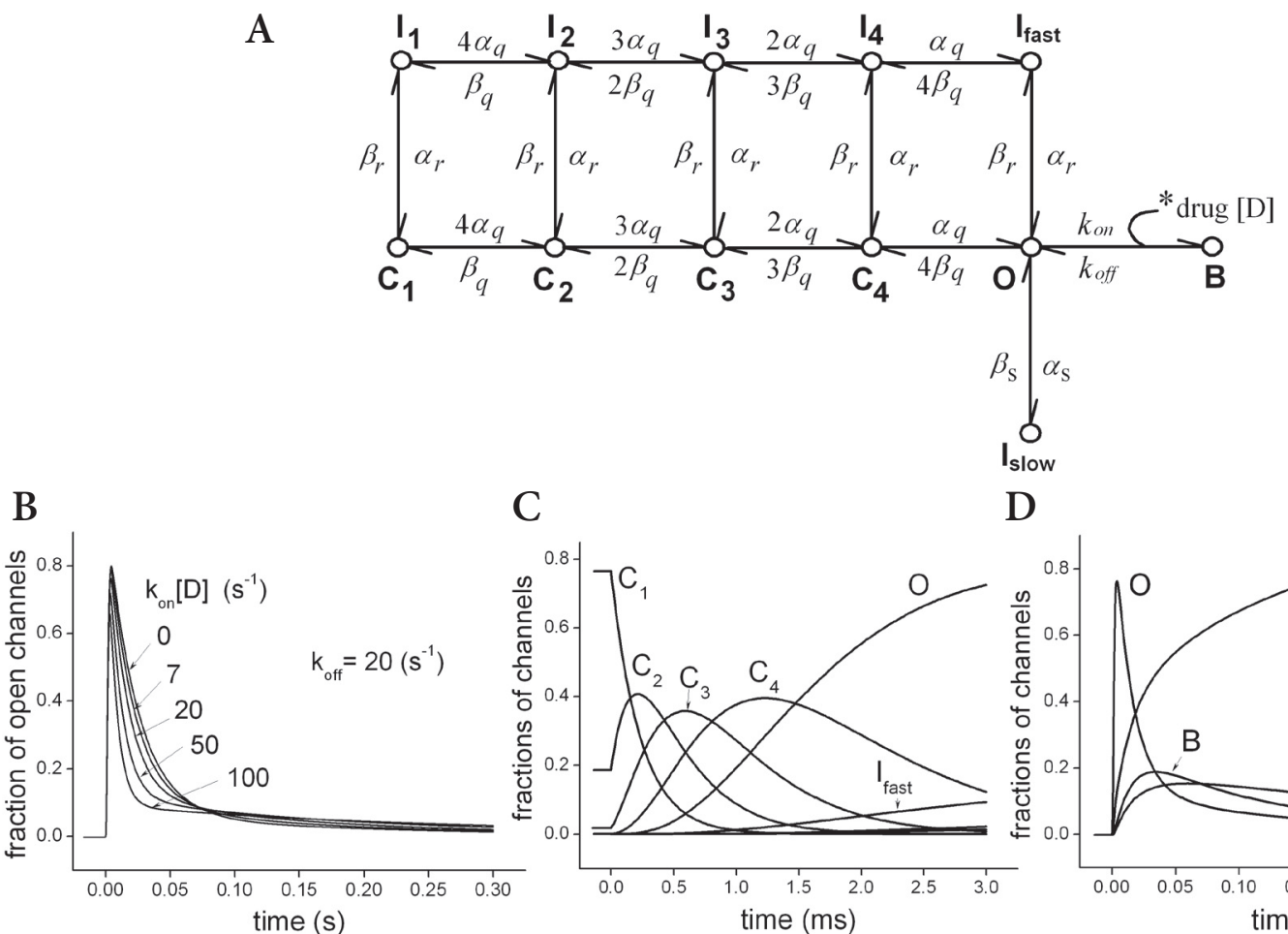

$\mathrm{D}$

Figure 1. Model of $I_{t o}$ current kinetics. A. State diagram of $I_{t o}$ channel gating and open channel block. $C_{1}, C_{2}, C_{3}, C_{4}$, closed states; $I_{1}, I_{2}, I_{3}, I_{4}$, fast inactivated states (from closed states); $\mathrm{I}_{\text {fast }}$, fast inactivated state (from open state); $\mathrm{I}_{\text {slow }}$ slowly inactivated state; $\mathrm{O}$, open channel state; $\mathrm{B}$, blocked channel state; $[D]$, drug concentration. B. Superimposed fractions of open channels in response to a single voltage step from -120 to $+60 \mathrm{mV}$ at $k_{o f f}=20 \mathrm{~s}^{-1}$ and $k_{o n}[D]=0,7,20,50$ and $100 \mathrm{~s}^{-1}$. Simultaneous fractions of channels found in different channel states (at $k_{o f f}=20 \mathrm{~s}^{-1}$ and $k_{o n}[D]=20 \mathrm{~s}^{-1}$ ) in response to a single voltage step from -120 to $+60 \mathrm{mV}$ within the first $3 \mathrm{~ms}(\mathrm{C})$ and during the following $300 \mathrm{~ms}(\mathbf{D})$. 
Under the assumption of an approximately linear relationship between the drug concentration $[\mathrm{D}]$ and the reciprocal value of time constant of fast component of apparent inactivation $\tau_{b f}$ (Snyders et al. 1992; Sanchez-Chapula 1999), the rate constants of binding can be estimated from a formula:

$$
\frac{1}{\tau_{b f}}=k_{o f f}+k_{o n}[D]
$$

To evaluate the accuracy of the rate constants estimated in this way, we analysed the solution of the equations describing the state diagram in Fig. 1A in detail. After the activation has been completed, channels transit solely among open $(\mathrm{O})$, inactivated $\left(\mathrm{I}_{\text {fast }}, \mathrm{I}_{\text {slow }}\right)$ and drug-occupied (B) channel states (Fig. 1D). The time course of apparent inactivation could then be described by a reduced four-state model and a corresponding set of four first-order homogenous linear differential equations. In a preliminary study (see Appendix in Bébarová et al. 2005), we outlined the way to derive an improved formula enabling calculations of binding rate constants $\left(k_{o n}, k_{\text {off }}\right)$ from a threestate reduced model including only the fast inactivation:

$$
\frac{1}{\tau_{b f}}+\frac{1}{\tau_{b s}}-\frac{1}{\tau_{i}}=k_{o f f}+k_{o n}[D]
$$

The time constants $\left(\tau_{b f}, \tau_{b s}\right)$ on the left side of Eq. (2) could be estimated from the approximation of apparent inactivation by a sum of fast and slow exponential functions for several selected drug concentrations. The time constant $\tau_{i}$ resulted from approximation of inactivation by a single exponential in the absence of a drug.

In this study, we extended this approach including two time constants of inactivation in the absence of drug marked $\tau_{\text {if }}$ and $\tau_{i s}$. Under the drug effect, the apparent inactivation is to be approximated by three exponentials resulting in estimation of time constants $\tau_{b f}, \tau_{b s}$ and $\tau_{b u s}$ :

$$
\frac{1}{\tau_{b f}}+\frac{1}{\tau_{b s}}+\frac{1}{\tau_{b u s}}-\frac{1}{\tau_{i f}}-\frac{1}{\tau_{i s}}=k_{o f f}+k_{o n}[D]
$$

First, the time constants were obtained analytically from reciprocals of the roots of characteristic equation of the system of 12 linear differential equations describing the response of $I_{t o}$ channels to a step of membrane voltage. One root of the characteristic equation was zero, eleven roots were nonzero. Absolute values of their reciprocals are time constants that appear in the sum of exponentials describing simulated time courses of the fractions of channels in individual states. Eight short time constants (in the order of $0.1-1 \mathrm{~ms}$ ) were related to $I_{t o}$ activation, the remaining three (in the order of 10-100 ms) corresponded with the course of apparent inactivation (constants $\tau_{b f}, \tau_{b s}$ and $\left.\tau_{b u s}\right)$. For verification, the time constants $\tau_{b f}, \tau_{b s}$ and $\tau_{b u s}$ were obtained also from numerical solution of the equation system. They were estimated in the same way as in the experiments. The simulated fractions of open channels in the course of apparent inactivation were approximated by a sum of exponential

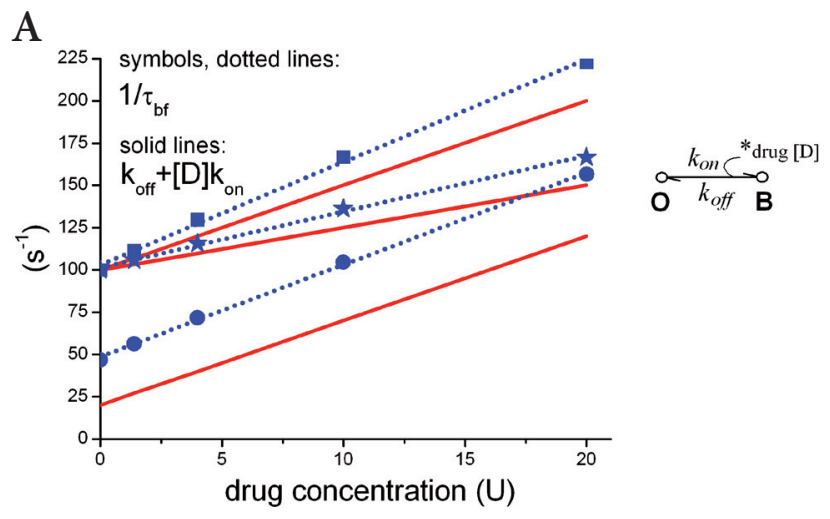

B

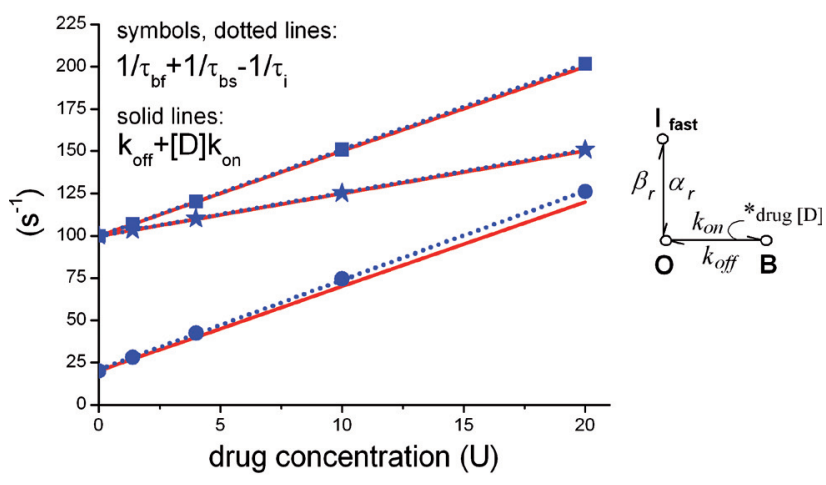

C

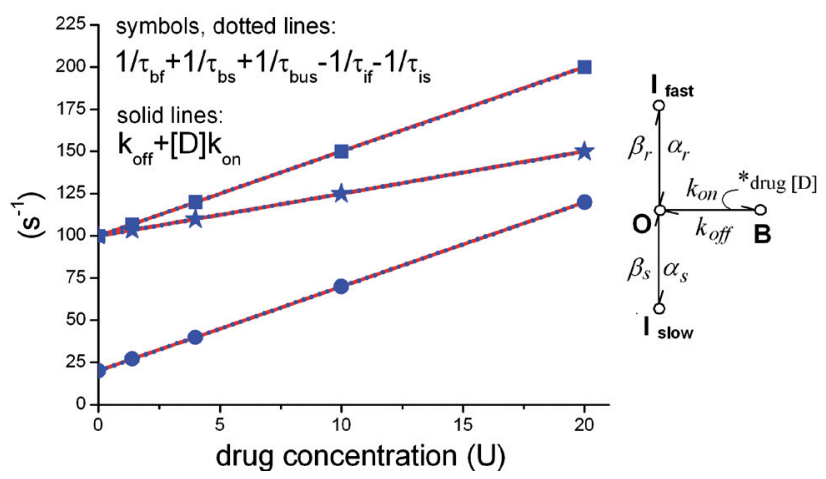

Figure 2. Evaluation of the drug binding rate constants $k_{\text {off }}$ and $k_{\text {on }}$ from the time course of apparent inactivation of $I_{t o}$. Comparison of the accuracy of the rate constants estimated according to the formulas Eq. 1 (A), Eq. 2 (B), Eq. 3 (C). Solid lines represent reference linear concentration dependence of the expression $k_{o f f}+k_{\text {on }}[D]$ constructed from the pairs of $k_{\text {off }} k_{\text {on }}$ values inserted into the complete model. Symbols approximated with dotted lines represent result of concentration dependence of the terms in the left sides of formulas (1), (2), (3) corresponding to experimental data. Rate constant $k_{\text {off }}$ is determined as the intersection of each line with the ordinate, $k_{\text {on }}$ as the slope of the line. The pairs of values of rate constants selected for comparison: $-k_{o f f}=100 \mathrm{~s}^{-1}, k_{o n}=5 \mathrm{U}^{-1} \mathrm{~s}^{-1}$; * $k_{o f f}=100 \mathrm{~s}^{-1}$, $k_{o n}=2.5 \mathrm{U}^{-1} \mathrm{~s}^{-1} ; \bullet k_{\text {off }}=20 \mathrm{~s}^{-1}, k_{\text {on }}=5 \mathrm{U}^{-1} \mathrm{~s}^{-1} ; \mathrm{U}$, arbitrarily selected unit of drug concentration $[D]$. The simplified kinetic diagrams right to the graphs indicate processes following full activation of $I_{t o}$ channels taken into account in the models under comparison. 
functions using the least-squares method. The results fitted well with those obtained using the analytical approach.

We took advantage of mathematical modelling which gives the possibility of comparing the real values of binding rate constants incorporated into the model with those evaluated from measurable parameters (i.e. the time constants obtained from a fit of the time course of apparent inactivation to a sum of exponential functions).

The solid lines in Fig. 2 show a linear concentration dependence of the common term in the right side of Eqs. (1), (2), (3) corresponding to several representative values of $k_{\text {off }}$ and $k_{o n}$. The intersection of each line with the ordinate determines $k_{\text {off while the slope determines }} k_{\text {on }}$. The values marked by symbols are calculated from the left sides of Eqs. (1), (2), (3) for selected drug concentrations. The dotted lines resulting from the linear regression of simulated experimental data enable the estimation and comparison of errors in $k_{\text {off }}$ and $k_{\text {on }}$ evaluated from the models described by Eqs. (1), (2), and (3) and illustrated in panels A, B, and C, respectively. To simulate the effect of slow component of inactivation corresponding to the maximum magnitude observed (Bébarová et al. 2009), the value of $\alpha_{s}$ was doubled (increased to $16 \mathrm{~s}^{-1}$ ).

Actually, the presented models reflect a description of the processes following full activation of $I_{t o}$ channels, as illustrated in Fig. 2 to the right of the graphs. The model described by Eq. (1) neglects transitions to inactivated states, model (2) neglects slow inactivation, while model (3) considers both fast and slow inactivation.

Fig. $2 \mathrm{~A}$ shows that calculations according to the model (1) tend to overestimate both $k_{\text {off }}$ and $k_{o n}$. At higher values of $k_{\text {off }}$ the degree of overestimation of $k_{\text {off }}$ decreases while overestimation of $k_{o n}$ increases. The accuracy of calculated binding constants was substantially enhanced when three time constants with respect to formula (2) were used for the reconstruction (Fig. 2B). The error was practically negligible when the model (3) was applied (Fig. 2C).

The model described by formula (2) was used in our earlier experimental work focused on the effects of antiarrhythmic drug ajmaline on $I_{t o}$ in rat ventricular myocytes (Fig. 2 in Bébarová et al. 2005). The values of $k_{\text {on }}$ and $k_{\text {off }}$ estimated from this model were $4.57 \times 10^{6} \mathrm{~mol}^{-1} \cdot 1 \cdot \mathrm{s}^{-1}$ and $20.12 \mathrm{~s}^{-1}$, respectively, while the values evaluated from the simplified model (1) were overestimated: $k_{\text {on }}$ by $5 \%$ and $k_{\text {off }}$ even by $90 \%$.

The accurate assessment of the kinetic parameters of drug-channel interactions became an essential part of the up-to-date evaluation of the effects of antiarhythmics and side cardiac effects of clinically used drugs. Thus, it would be useful in future work to extend the presented analysis to the drugs that inhibit channels in other than the open state.

Acknowledgement. This work has been supported by the grant project MSM0021622402 from the Ministry of Education, Youth and Sports of the Czech Republic.

\section{References}

Bébarová M., Matejovič P., Pásek M., Šimurdová M., Šimurda J. (2005): Effect of ajmaline on transient outward current in rat ventricular myocytes. Gen. Physiol. Biophys. 24, 27-45

Bébarová M., Matejovič P., Pásek M., Jansová D., Šimurdová M., Nováková M., Šimurda J. (2009): Effect of antipsychotic drug perphenazine on fast sodium current and transient outward potassium current in rat ventricular myocytes. Naunyn Schmiedebergs Arch. Pharmacol. 380, 125-133 http://dx.doi.org/10.1007/s00210-009-0420-1

Caballero R., Pourrier M., Schram G., Delpon E., Tamargo J., Nattel S. (2003): Effects of flecainide and quinidine on Kv4.2 currents: voltage dependence and role of S6 valines. Br. J. Pharmacol. 138, 1475-1484 http://dx.doi.org/10.1038/sj.bjp.0705199

Casis O., Sánchez-Chapula J. A. (1998): Mechanism of block of cardiac transient outward K+ current (Ito) by antidepressant drugs. J. Physiol. (London) 32, 527-534

Castle N. A., Slawsky M. T. (1993): Characterization of 4-aminopyridine block of the transient outward $\mathrm{K}+$ current in adult rat ventricular myocytes. J. Pharmacol. Exp. Ther. 264, 1450-1459

Christé J., Šimurda J., Šimurdová M. (1995): Use-dependent features of 4-aminopyridine block of transient outward current in rat ventricular myocytes. Gen. Physiol. Biophys. 14, 75-89

Clark R. B., Sánchez-Chapula J. A., Salinas-Estefanon E., Duff H. J., Giles W. R. (1995): Quinidine-induced open channel block of $\mathrm{K}+$ current in rat ventricle. Br. J. Pharmacol. 115, 335-343

Hille B. (1977): Local anesthetics: hydrophyllic and hydrophobic pathways for the drug-receptor reaction. J. Gen. Physiol. 69, 497-515 http://dx.doi.org/10.1085/jgp.69.4.497

Jahnel U., Klemm P., Nwrath H. (1994): Different mechanisms of the inhibition of the transient outward current in rat ventricular myocytes. Naunyn Schmiedebergs Arch. Pharmacol. 349, 87-94 http://dx.doi.org/10.1007/BF00178211

Patel S. P., Parai R., Parai R., Campbell D. L. (2004): Regulation of $\mathrm{Kv} 4.3$ voltage-dependent gating kinetics by KChIP2 isoforms. J. Physiol. (London). 557, 19-41 http://dx.doi.org/10.1113/jphysiol.2003.058172

Patel S. P. and Campbell D. L. (2005): Transient outward potassium current, ,Ito', phenotypes in the mammalian left ventricle: underlying molecular, cellular and biophysical mechanisms. J. Physiol. (London) 569, 7-39 http://dx.doi.org/10.1113/jphysiol.2005.086223

Sanchez-Chapula J. A.(1999): Mechanism of transient outward $\mathrm{K}+$ channel block by disopyramide. J. Pharmacol. Exp. Ther. 290, 515-523

Snyders D. J., Knoth K. M., Roberds S. L., Tamkun M. M. (1992): Time-, voltage-, and state-dependent block by quinidine of a cloned human cardiac potassium channel. Mol. Pharmacol. 41, 322-330

Wagner M., Riepe K. G., Eberhardt E., Volk T. (2010): Open channel block of the fast transient outward $\mathrm{K}+$ current by primaquine and chloroquine in rat left ventricular cardiomyocytes. Eur. J. Pharmacol. 547, 13-20 http://dx.doi.org/10.1016/j.ejphar.2010.08.007

Wettwer E., Himmel H. M., Amos G. J. Li Q., Metzger F., Ravens U. (1998): Mechanism of block by tedisamil of transient outward current in human ventricular subepicardial myocytes. Br. J. Pharmacol. 125, 659-666 http://dx.doi.org/10.1038/sj.bjp.0702110

Received: October 31, 2011

Final version accepted: February 15, 2012 